\title{
Research on Integrated Conflict Management in the Collaborative Design
}

\author{
Zhongxian $\mathrm{Bao}^{1}$, Pengshou $\mathrm{Xie}^{1}$ and Mao Wang ${ }^{2}$ \\ ${ }^{1}$ School of Computer and Communication, Lanzhou University of Technology, Lanzhou 730050, China \\ ${ }^{2}$ School of Science, Lanzhou University of Technology, Lanzhou 730050, China
}

\begin{abstract}
It is limited to resolve conflict with single conflict resolution method in cooperative design. The conflict classification, the conflict detection and conflict resolution technology were discussed and an integrated conflict management model is developed in this paper. In this model, according to the types of conflicts, system can select one or more appropriate conflict resolution to realize conflict resolution efficiently.
\end{abstract}

Keywods-collaborative design; integrate; conflict resolution; conflict management

\section{INTRODUCTION}

In the process of product collaborative design, conflicts are everywhere and inevitably, As the conflict continues to be resolved, the design will be gradually improved. in a sense, the process of collaborative design is a process of conflict and resolution. Many scholars have carried a lot of research in the conflict detection and conflict resolution, many conflict detection and conflict resolution method is found, got good effect, but each method has certain application area. In this paper, based on the study of conflict detection and conflict resolution method, integrated Conflict management model in the Collaborative Design is developed to realize conflict resolution efficiently [1]- [4] .

\section{CONFlict Detection Method}

In the collaborative design process, conflicts have occurred must be detected in effective means, to ensure the subsequent normal design can be done in a normal conditions. Collision detection is a key technology of collaborative design , it was studied from different angles and in different methods.

\section{A. Conflict Detection Method Based on Integrated Petri Net}

A conflict detection method based on integrating Petri net is studie were proposed to detect the conflicts. Several algorithms for $\mathrm{d}$ in [5], collaborative design system is a multi agent system, the relationship between agents generally have serial relationship, parallel relationship, direct cause and effect relationship, indirect cause and effect relationship, and the coupling relationship. In order to simplify the conflict detection model, agents is divided reasonably at the beginning of the system design to avoid the coupling between agents. According to the relationship between the agents in the collaborative design environment, the Petri net of all description agents can be integrated to form an integrated Petri net with an output library, a collision detection model is built.

\section{B. Matrix Search for Collision Detection}

Wang Ke, Mingqing Wang proposed matrix searching method to carry on the conflict detection in [6], according to concurrent execution ,many matrix were design ,such as resource allocation and sharing of the collaborative groups , design group matrix, owning resource matrix, allocated resource matrix, the total allocated matrix of various types resources and need to apply resources matrix in each design group still need to apply. In the process of collaborative design, by means of the matrix search ,conflict is detected.

\section{Collision Detection Method Based on Constraint}

Meng Xiuli et al. in [7] proposed conflict detection method based on constraint. In mechanical product collaborative design starting from the point of constraint satisfaction, conflict detection problems are discussed, the genetic algorithm is applied to solve the constrained satisfaction problems, and the conflict detection system architecture based on constraint is proposed. Jia Yanbo, Sun Lijuan, et al. considers the conflict problem at the constrain-based aspect, and transforms the conflict detection to the constraint satisfaction problem of the constraint network of the collaborative design and applies the genetic simulated annealing algorithm (SAGA) t o solve the problem [8]. Zhao Huishe et al from the view of constraint constraint satisfacation, a hierarchical model , which includes product level, component level, part level and domain level,was present $d$ for the management of constraint network . Constraint verification and interval propagation algorithms generation of conflict negotiation suggestions based on backtracking and constraint relaxation strategies were put forward in [9]. Sheng Buyun et al give out the technology of conflict detection based on relational database in [10]. there are many scholars from the perspective of constraint point of view to explore the conflict detection problem.

\section{Conflict Detection Method Based on Geometric}

Wang Dayong et al proposed conflict detection method based on geometry class in [11]. Jiang Zhong Shang et al proposed a geometry level conflict detection strategy as well as its timing sequence scheme in [12], and application in Collaborative CAD system.

\section{Conflict RESOlution Method}

In conflict resolution, the scholars at home and abroad have done a lot of research work, the conflict resolution were studied from many aspects, such as the work flow of product design, 
design knowledge representation and storage and reasoning process, the decision space of design and so on. The constraint resolution method can be rule based conflict resolution method , constraint based conflict resolution method, case based conflict resolution method, based on the integration of the conflict resolution method, etc.

\section{A. Rule Based Conflict Resolution Method}

The conflict rules are summed up into the form of knowledge base, in support of the reasoning mechanism, help designers to resolve the conflict. rule based conflict resolution is a essential simple and effective conflict resolution method in collaborative design. Zhao Yang et al in [13], Ma Jun et al in [14] applied rule based conflict resolution method in various way. In practice, due to the existence of a variety of domain knowledge, the method of inference rules, the collaborative group perhaps is lack of understanding in other areas of knowledge, so conflict is happened. if domain knowledge is formed a knowledge base by means of rough set, rules and so on, in the reasoning mechanism conflict can be resolution.

\section{B. Constraint Based Conflict Resolution Method}

With constraint based conflict resolution method, in the several ways of showing the complex constraints, canceling the conflict, ignoring constraints and relaxing the constraints, the conflict can be resolved. In the collaborative design process, the design groups through the constraint to describe their mutual restraint, mutual dependent relationship, so the conflict relaxation can be used when the constraint occurs, constraint relaxation method to eliminate conflict by relaxing some constraints or ignoring the secondary constraints to achieve the purpose of conflict resolution. Li Yuan et al in [15] given it application in multi computer cooperative task. the mission plan is transformed into scheduling model with multi-capacitated resources, and the definition of resource conflicts and rules for conflict judgment are given based on the model's temporal properties a resource conflict detection algorithm is presented based on the minimal critical sets, which makes use of the properties of overlaps between temporal bounds, and the resource conflicts are detected and resolved as particular cliques in connected graph. Jiang Yiling et al in [16] focusing the process conflict of automotive conFigureuration, took mathematical model, customized requirements and knowledge as conFigureuration items, and then constraint model was built . Conflict effect weight was proposed, and constraint loosen based conflict strategy was used in constraint level model. Zhao Huishe discussed the backtracking and constraint relaxation strategies of conflict negotiation in [9], gives several suggestions of negotiation algorithm.

\section{Case Based Conflict Resolution Method}

On based constraint based conflict resolution method, by means of showing the complex constraints, canceling the conflict, ignoring constraints and relaxing the constraints, by finding examples similar to the current conflict, a conflict outline solution is provided rapidly, and it is further modified and perfected. Meng Xiuli et al in [17] discussed this method in the machine tool products collaborative design, described the conflict knowledge with frame structure an transforms the structure based on relational database; they proposed a case search model which is based on primary feature an minor feature, established a flexible case retrieval mechanism model, provided a case base maintenance functions and typical conflict of self-learning.

\section{Integration Based Conflict Resolution Method}

Because of the conflicts in the process of collaborative design differ from one another, conflict causes and conflict forms is different from each other, using single conflict resolution method to solve complex conflicts is hard to get the ideal result. Integration based on conflict resolution method is proposed. According to conflict types, one or more conflict resolution is selected to resolve the conflict. Li Xiang et al proposed the integrated thought in [18], Meng Xiuli et al also discussed the integrated conflict resolution method in [17].

\section{CONFLICT Modeling}

To describe and to manage the conflict of collaborative design process effective, to complete the conflict classify and resolve it, building conflict model is urgently need. Reference [19], an object oriented conflict modeling is defined as follows.

class CMember $\{\ldots\}$; / / Collaborative group class

class CSet $\{\ldots\} ; / /$ Constraint set class

class CConflict/ / Conflict class

\{

int conflictid ; // Unique identity of conflict

i nt conflictlevel ; //Conflict level

i nt conflicttype ;//Conflict type

CTimeconflictcreatetime ; //Occurrence time of conflict

int resolution status ;// Conflict resolution status

string conflictrepresentation ( ) ; //Conflict representation

CMember *p_involvedmember ; // Design group involved in conflict

CSet *p_violationset ; // Conflict set violated in conflict

$\cdots$

\} ;

Class CConflictManagement //Conflict management class

$\{\ldots$

public :

void CreateConflict ( ) ; // Create a conflict

void RegisterConflict ( ) ;// Record a conflict

void ResolutionHistory ( ) ; //History record of conflict resolution

void UpdateConflict ( ) ;// Update a conflict

void DeleteConflict ( ) ;// Delete a Conflict

\} ; 


\section{Classification OF CONFLiCts}

Conflict is inevitable in the process of collaborative design. Conflict is everywhere in collaborative design. According to [4][5], The conflicts in product design are summarized into the following categories:

(1) Plan conflict: Plan conflict means the design delay or even design fail leaded by poor planning or an inappropriate intervention time of different designers. It also means the actual process of product development is not consistent with the process model of collaborative design, the current design is delayed.

(2) Knowledge conflict: Knowledge conflict means that the rules in different areas is difficult to meet each other. For example, the rules, facts, heuristic search knowledge and the constraint resolution are inconsistent, incomplete and incompatible.

(3) Design conflict: Design conflict means relationship of mutual antagonism, mutual conflict in product, it is divided into design results conflict and design objectives conflict. The design results conflict shows on antagonism and conflict relationship between design schemes or design attributes, it cannot satisfy the function constraint or assembly constraints or manufacturing constraints and so on. The design goals shows on that design schemes or design attributes cannot satisfy the function target, manufacturing target, assembly target and the other various design target.

(4) Resource conflict. It is caused by the limited resources, mainly refers to the conflict of auxiliary design resources. Auxiliary design resources include human resources, hardware resources, information resources and other non directly using in the product design.

(5) Process conflict: It means the conflict caused by the time contradictory and the information contradictory in the product development process. Process conflict showing on time is tardiness conflict, that a task has not been completed according to plan it should be completed, and it lead to the following task cannot start; Process conflict showing on information is the absence of conflict, that a task cannot start because of waiting output information of the cannot previous tasks . The process conflict causes the whole design delay.

(6) Data conflict: this is the conflicts caused by the problem of data consistency. the design data is inadequate to the requirements of two or more designers at the same time, and the system is lack of coordination with the same data access of two or more designer groups.

\section{INTEGRATED CONFLICT MANAGEMENT MODEL}

Effective conflict management is safeguard that collaborative design can be done smoothly and efficiently. Conflict management is not a simple conflict solve process, it is overall coordination and management activities of collaborative design process , conflict is clue in this activities. Based on the analysis of a large number of conflict detection and conflict resolution methods, to avoid the limitations of single conflict resolution method, an integrated conflict management model is built for the collaborative design as shown in Figure 1. The model includes three modules as support module design, conflict management module and user module.

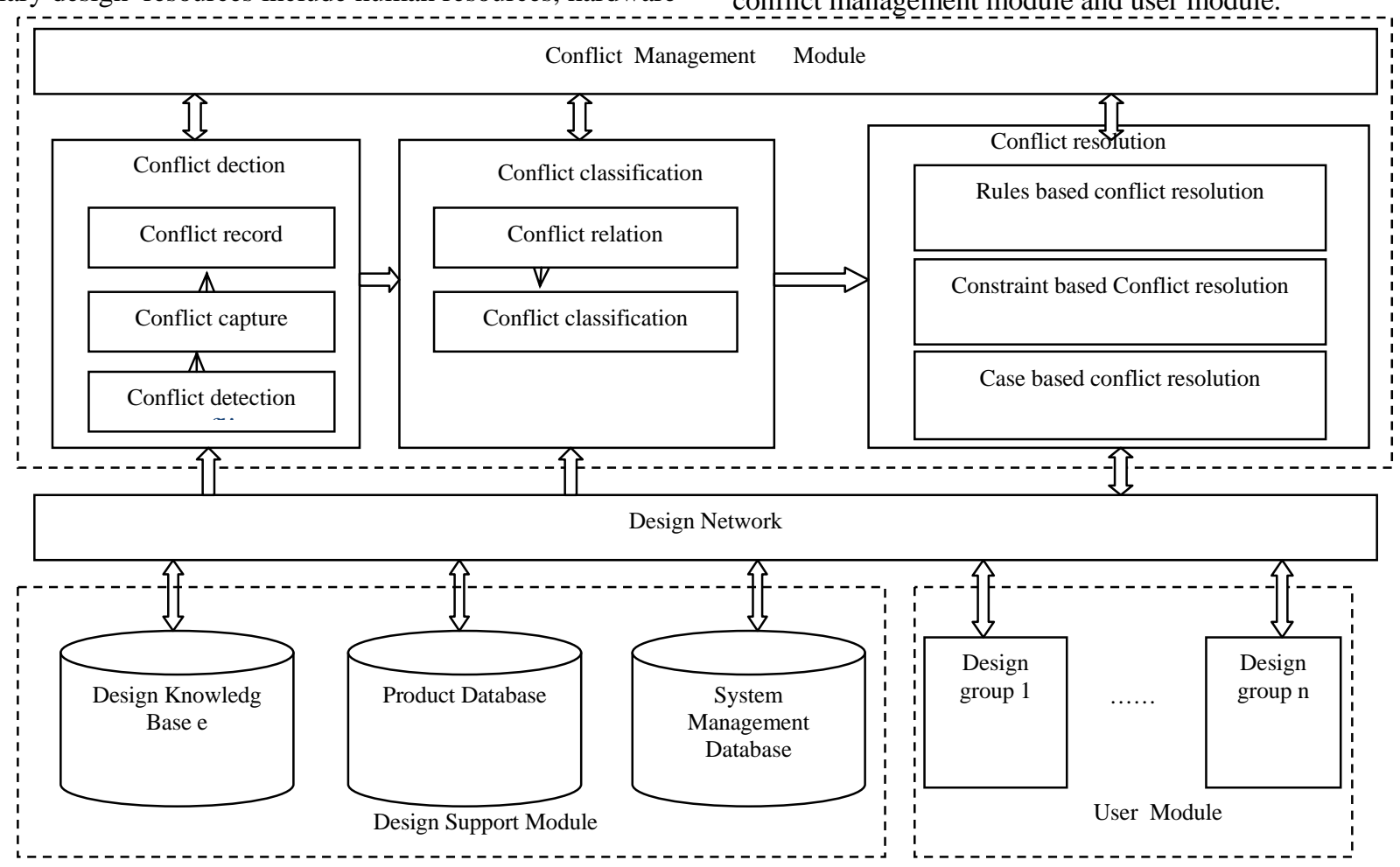

FIGURE.I. INTEGRATED CONFLICT MANAGEMENT MODEL 


\section{A. Design Support Module}

Design support module includes the design knowledge base, the product database, the system management database. The design knowledge base is used to store all kinds of knowledge data required for product design, it includes constraint data, conflict data, case data, rule data and so on.

\section{B. User Module}

The user module can call support module design and conflict management module by Internet Intranet or the client, so the conflict resolution is realized in the process of design

\section{Conflict Management Module}

Conflict management module can realize conflict management in whole lifecycle of collaborative design. This module includes three submodules, it can realize the conflict management from conflict detection, Conflict capture to conflict resolution and in the module there stores various functional models for the conflict management. The conflict detection method, including the conflict detection method based on Petri net, matrix search method, conflict detection method based constraint, the geometric level conflict detection and other conflict detection methods. Conflict resolution methods include rule based conflict resolution method, constraint based conflict resolution method, case based conflict resolution method, and so on.

\section{CONCLUSION}

In view of the complexity and diversity of the conflict in the collaborative design of mechanical products, the conflict management is studied from the perspective of the whole life cycle. Firstly, the causes of conflict are analyzed, and the conflict is classified in detail. Then has conducted the research to the conflict detection technology, including integrating Petri net conflict detection method, matrix search method, based on constraint conflict detection technology, based on geometry level conflict detection and conflict detection method based on. The rule based conflict resolution method is studied, which is based on the constraint change conflict resolution method, the case based conflict resolution method, and the integration based conflict resolution method and so on. Considering mechanical product collaborative design conflict of the complexity and diversity, to avoid the limitation of single conflict resolution method, classification and archiving of conflicts in the process of design, adopt appropriate conflict resolution method according to the type of conflict, design a integrated conflict management model based on.

\section{ACKNOWLEDGMENT}

This work is supported by the Natural Science Foundation of Gansu Province, China(Grant No. 1212RJZA035)

\section{REFERENCES}

[1] J.Ma. Collaborative and Decision Technology for Innovative Design [M]. Beijing: Science Press,2008

[2] [2]JH.Li. Computer Supported Cooperative Work [M]. Beijing: Machinery Industry Press, 2010
[3] [3] Y.Tang,GF.Ji and J.Zhu. Collaborative Software Technology and Application [M]. Beijing: Machinery Industry Press, 2010

[4] W.Liu,L.Li,F.Zhou,YF.WANG and Y.Yin. On Conflic t Management in Collaborative Design. Mechanical Science and Technology. 2003 Vol 22 (7): 192

[5] M.Chen. Design of Conflict Detection Model in the Collaborative Design. Computer Era. 2003. 7:3

[6] K. Wang, MQ. Wang. Research on Conflict Inspection and Conflict Elimination in Collaborative Design.Journal of Jiangsu University of Science and Technology(Natural Science Edition). 2007.3:63

[7] XL.Meng, H.Yi, ZH.Ni and Y.Liu. Research on technologies of constraint-based conflict detection in collaborative design. Computer Integrated Manufacturing Systems.2004.11:1426

[8] YB.Jia,Sun. Research of the Conflict Detection of the Collaborative Design. Journal of Harbin University of Science and Technology Univ. 2007.6:7

[9] HS.Zhao,L.Tian,BS.Tong.Constraint-Based Conflict Detection and Negoiation in Collaborative Design. Computer Integrated Manufacturing Systems. 2002.11:896

[10] BY.Sheng, ZJ.Lin and YF.Ding.Research on Technologies of RDB-based Conflict Detection in Collaborative Design. Mechanical Design \& manufacture.2006.9:66

[11] DY.Wang and WD.Jin. Conflict detection algorithmin collaborative design. Computer Applications. 2007.3:650

[12] JZ.Shang,L.Tang,FL.Meng and ZR.Luo. Research on the Strategy of Geometr y Level Conflict Detection Based on the Concurrent Timing Sequence.Journal of National university of Defense Technology. 2004.5:90

[13] Y.Zhao and H.Liu. Research on Rules Inference -based Conflict Resolution Systemfor Collaborative Design. Application Research of Computer. 2006.1:54

[14] J.M and XM.Ji.Implementation of Conflict Resolution for Process Decision Based on Rough Theory. Journal of Computer-aided Design \&Computer graphics .2005.3:600

[15] Y.Li, H.Peng and LC.Shen. Constraint satisfaction based resource conflicts detection and resolution in collaborative mission planning. Systems Engineering and Electronics 2009.4:868

[16] YL. Jiang, J. Hu, WM. Wang, J. Qi and YH. Peng. Conflict Resolution in the Automotive ConFigureuration Process Based on Constraints Network. Journal of Shanghao Jiaotong University.2009.1:119

[17] XL.Meng,XD.Han and J.Cao. Research on Case-based Conflict Resolution System in Collaborative Design of Machine Tools. Manufacturing Information Enginnering of China.2005.10:109

[18] [18]X. Li, GX. Yuan, XH. Zhou and XY. Ruan. Research on Integration-Based Conflict Resolution System in Collaborative Design. Computer Integrated Manufacturing Systems.2000.8:61

[19] XL.Meng,XD.Han and J.Cao. Research on integrated conflict resolution system in collaborative design. Manufacturing Information Enginnering of China. 2006.3:32 